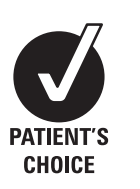

CHOICE

\title{
Occurrence and risk factors for apathy in Parkinson disease: a 4-year prospective longitudinal study
}

\author{
K F Pedersen, ${ }^{1,2}$ G Alves, $^{1,2}$ D Aarsland, ${ }^{1,3,4}$ J P Larsen ${ }^{1,2,4}$
}

\begin{abstract}
${ }^{1}$ The Norwegian Centre for Movement Disorders, Stavanger University Hospital, Stavanger, Norway; ${ }^{2}$ Department of Neurology, Stavanger University Hospital, Stavanger, Norway;

${ }^{3}$ Department of Psychiatry, Stavanger University Hospital, Stavanger, Norway; ${ }^{4}$ Institute of Clinical Medicine, University of Bergen, Bergen, Norway
\end{abstract}

Correspondence to: Dr K F Pedersen, The Norwegian Centre for Movement Disorders,

Stavanger University Hospital,

PO Box 8100, N-4068

Stavanger, Norway:

kenfrp@online.no

Received 9 December 2008 Revised 3 February 2009 Accepted 5 February 2009

\section{ABSTRACT}

Background: Apathy is a common but under-recognised behavioural disorder associated with depression and cognitive impairment in patients with Parkinson disease (PD). However, the longitudinal course of apathy in PD has not been studied.

Objective: To examine the occurrence of and risk factors for apathy over time in a representative sample of patients with PD.

Methods: A sample of 139 patients was drawn from a population-based prevalence study of PD in Rogaland County, Western Norway. Apathy was measured with the Neuropsychiatric Inventory, using a composite score $\geqslant 4$ to indicate clinically significant apathy. Additional measurements included standardised rating scales for parkinsonism, depression and cognitive impairment. A follow-up evaluation was carried out in 79 patients $(78.2 \%$ of the survivors) 4 years later.

Results: Of the 79 patients included in this study, 29 patients (36.7\%) had never had apathy, 11 (13.9\%) had persistent apathy, and a further 39 (49.4\%) developed apathy during follow-up. At follow-up, patients with apathy were more frequently depressed and demented than never-apathetic patients. Dementia at baseline and a more rapid decline in speech and axial impairment during follow-up were independent risk factors for incident apathy.

Conclusions: Apathy is a persistent behavioural feature in PD with a high incidence and prevalence over time. Progression of motor signs predominantly mediated by non-dopaminergic systems may be a useful preclinical marker for incident apathy in PD.

Apathy is a common but frequently under-recognised neuropsychiatric disorder in patients with Parkinson disease (PD) ${ }^{1}$ that is attributed to basal ganglia pathology and disturbances in frontalsubcortical connections. ${ }^{2}$ Cross-sectional studies have shown that apathy is associated with depression, cognitive deficits, a decrease in performing activities of daily living ${ }^{3-5}$ and possibly motor severity. ${ }^{6}$ However, the frequency and clinical correlates of apathy in $\mathrm{PD}$ over time have not yet been studied.

Here we report on the occurrence and risk for apathy with emphasis on motor, depressive and cognitive symptoms in a 4-year prospective population-based longitudinal cohort study of patients with prevalent $\mathrm{PD}$.

\section{METHODS}

\section{Subjects}

One-hundred and thirty-nine patients with PD from an ongoing prospective community-based study of PD in Rogaland County, Western
Norway, ${ }^{7}$ were invited during 1996 and 1997 to participate in the baseline evaluation of the present study and completed a comprehensive neurological, psychiatric and neuropsychological assessment after having provided written informed consent. ${ }^{4} \mathrm{~A}$ total of 79 patients $(78.2 \%$ of the survivors with $\mathrm{PD}$ ) were reassessed 4 years later using the same evaluations as those performed at baseline (50 patients had died, and 10 refused to participate). There were no significant differences in age, gender or education between non-participants and patients included at follow-up. In 22 cases from the study cohort who have come to autopsy so far, the findings were compatible with PD in all cases. ${ }^{8}$ The study was approved by the Regional Committee for Medical Research Ethics at the University of Bergen, Norway.

\section{Assessment}

The Unified Parkinson Disease Rating Scale (UPDRS) motor part ${ }^{9}$ and the Hoehn and Yahr stage $^{10}$ were administered when patients were in their best on state. The total UPDRS motor score (range 0-108) was divided into six motor domains (speech, facial expression, tremor, rigidity, bradykinesia and axial impairment) based on the cardinal clinical manifestations of PD. ${ }^{11}$ These six motor domains were grouped into two subscores that represented predominantly dopaminergic (subscore A: tremor, rigidity, bradykinesia and facial expression; range 0-88) and non-dopaminergic (subscore B: speech and axial impairment; range $0-20)$ deficiencies. ${ }^{11}$ Due to the variety of dopaminergic treatment, a daily levodopa equivalent dose was estimated, as previously described..$^{12}$

Dementia was diagnosed according to the Diagnostic and Statistical Manual of Mental Disorders, Third Edition, Revised (DSM-III-R) criteria $^{13}$ based on a care-giver-based interview, cognitive rating scales including Mattis Dementia Rating Scale ${ }^{14}$ and Mini-Mental State Examination (MMSE), ${ }^{15}$ and neuropsychological tests, as described in detail previously. ${ }^{16}$

Apathy was measured with the Neuropsychiatric Inventory (NPI), ${ }^{17}$ using a composite apathy score (severity multiplied by frequency) of $\geqslant 4$ indicating clinically significant apathy, as recommended in $\mathrm{PD} .{ }^{18} \mathrm{~A}$ high interrater reliability of the NPI apathy subsection has been demonstrated in PD. ${ }^{4}$

Major depression was diagnosed by a psychiatrist on the basis of a clinical interview according to DSM-III-R criteria. The Montgomery and Aasberg Depression Rating Scale (MADRS) ${ }^{19}$ was used to evaluate severity of depressive symptoms, and to diagnose minor depression when only sad mood 
was present (item 1 or 2 with a cut-off score $\geqslant 2)^{20}$ in addition to at least one other symptom of depression (with cut-off score $\geqslant 2){ }^{21}$ Both patients with major and minor depression were classified as being depressed. The NPI, dementia interview, cognitive and depression assessments were performed blind to the motor evaluation.

\section{Statistical analysis}

Mann-Whitney, Kruskal-Wallis tests and $\chi^{2}$ tests or Fisher exact tests were used for comparisons as appropriate. Wilcoxon signed rank tests were used to compare differences between paired groups over time. Binary logistic regression analyses with forward stepping (likelihood ratio method) were performed, with the presence or absence of apathy as the dependent variable. SPSS version 15.0 was used for the analyses. Twotailed $p$ values $<0.05$ were considered statistically significant.

\section{RESULTS}

\section{Development of apathy}

Demographic and clinical characteristics at study entry are provided in table 1.

The majority of patients (93\%) were in Hoehn and Yahr stage 2 or higher. At baseline, 11 of 79 patients (13.9\%) were apathetic, whereas 68 (86.1\%) were not. At the 4-year study visit, all patients with apathy at baseline remained apathetic, and a further 39 subjects (49.4\%) had developed apathy during the study period. Twenty-nine patients (36.7\%) were without apathy at both baseline and follow-up.

\section{Motor, cognitive and neuropsychiatric correlates of apathy}

Patient characteristics according to apathy status are presented in table 2 .

There were no significant differences between the three apathy groups regarding demographic (age, gender, education or disease duration) and treatment (dopaminergic, antidepressant, anxiolytic or antipsychotic medication) variables (data not shown). Compared with those who remained non-apathetic during the study period, patients with incident apathy were more frequently demented at baseline, and more frequently depressed and demented at follow-up. Compared with patients free from apathy during the study period, patients with incident apathy at follow-up had a significantly more rapid increase in the UPDRS motor score, motor subscore B and MADRS score, as well as a more rapid decline in MMSE score.

Patients with persistent apathy during the observation period were more frequently depressed at baseline, and had a more pronounced increase in total UPDRS motor score and motor subscore A, than those with incident apathy at follow-up. Persistent apathy was not associated with incident depression or dementia at follow-up. There was no significant difference on NPI apathy scores between patients with persistent and incident apathy at follow-up.

\section{Risk factors of apathy}

In the regression analysis, we first entered age at baseline, changes in motor subscore $\mathrm{A}$ and $\mathrm{B}$, and changes in MADRS and MMSE scores as predictor variables for the presence or absence of apathy. An increase in motor subscore B was the only independent predictor of incident apathy in the model (odds ratio $1.345,95 \%$ CI 1.067 to $1.696, p=0.012$ ). Because a change in total UPDRS motor score was highly intercorrelated with changes in motor subscore A (Pearson $\mathrm{r}=0.980$ ) and $\mathrm{B}$ (Pearson $r=0.767$ ), only changes in motor subscores were included in the multivariate analysis. In a second step, we exchanged changes in MADRS and MMSE scores with presence of depression and dementia at baseline, while all other covariates remained unchanged, and found that both dementia at baseline (odds ratio $6.492,95 \%$ CI 1.281 to $32.898, \mathrm{p}=0.024$ ) and an increase in motor subscore B (odds ratio 1.268 , $95 \%$ CI 1.055 to 1.524 , $p=0.011$ ) had a significant effect. Both the Omnibus tests of

Table 1 Baseline demographic and clinical findings in patients with and without apathy

\begin{tabular}{|c|c|c|c|c|}
\hline & All patients & Apathy & No apathy & \\
\hline & $(n=79)$ & $(n=11)$ & $(n=68)$ & p Value \\
\hline Female, n (\%) & $44(55.7)$ & $7(63.6)$ & $37(54.4)$ & 0.746 \\
\hline Age in years (SD) & $72.0(8.3)$ & $73.4(8.4)$ & $71.8(8.3)$ & 0.533 \\
\hline Education in years (SD) & $9.3(3.3)$ & $9.0(2.7)$ & $9.3(3.4)$ & 0.733 \\
\hline Disease duration in years (SD) & $13.0(4.7)$ & $14.2(4.4)$ & $12.8(4.8)$ & 0.297 \\
\hline Total UPDRS motor score (SD) & $28.5(17.6)$ & $23.8(10.2)$ & $29.3(18.5)$ & 0.650 \\
\hline UPDRS motor subscore A (SD) & $18.4(12.3)$ & $14.5(6.9)$ & $19.0(12.9)$ & 0.436 \\
\hline UPDRS motor subscore B (SD) & $7.2(4.5)$ & $7.5(3.7)$ & $7.2(4.7)$ & 0.594 \\
\hline \multicolumn{5}{|l|}{ Hoehn and Yahr stage } \\
\hline Mean (SD) & $3.0(0.9)$ & $3.2(0.6)$ & $3.0(1.0)$ & 0.306 \\
\hline Range & $1.0-5.0$ & $2.5-4.0$ & $1.0-5.0$ & \\
\hline $\begin{array}{l}\text { Montgomery and Aasberg Depression Rating } \\
\text { Scale score (SD) }\end{array}$ & $5.4(6.7)$ & $13.3(7.3)$ & $4.2(5.6)$ & $<0.001$ \\
\hline Mini-Mental State Examination score (SD) & $25.5(6.4)$ & $21.1(6.7)$ & $26.2(6.1)$ & 0.011 \\
\hline Neuropsychiatric Inventory apathy score (SD) & $1.2(2.9)$ & $7.9(2.6)$ & $0.1(0.6)$ & $<0.001$ \\
\hline Depression, n (\%) & $16(20.3)$ & $6(54.5)$ & $10(14.7)$ & 0.007 \\
\hline Dementia, n (\%) & $23(29.1)$ & $7(63.6)$ & $16(23.5)$ & 0.012 \\
\hline Levodopa equivalent dose, mg daily (SD) & $658(387)$ & $630(415)$ & $663(386)$ & 0.456 \\
\hline Antidepressants, n (\%) & $14(17.7)$ & $3(27.3)$ & $11(16.2)$ & 0.370 \\
\hline Anxiolytics, n (\%) & $7(8.9)$ & $1(9.1)$ & $6(8.8)$ & 0.999 \\
\hline Antipsychotics, n (\%) & $9(11.4)$ & $3(27.3)$ & $6(8.8)$ & 0.084 \\
\hline
\end{tabular}

Data are expressed as mean (SD) or number (percentage of assessed patients); all available data included. Comparisons between patients with and without apathy: $\chi^{2}$ tests or Fisher exact tests when appropriate for categorical variables, Mann-Whitney tests for continuous variables. All p values are two-tailed.

UPDRS, Unified Parkinson Disease Rating Scale. 
Table 2 Neurological and psychiatric changes in patients with never apathy, incident apathy and persistent apathy during the 4-year observation period

\begin{tabular}{|c|c|c|c|c|}
\hline & $\begin{array}{l}\text { Never apathy } \\
(\mathrm{n}=29)\end{array}$ & $\begin{array}{l}\text { Incident apathy } \\
(\mathrm{n}=39)\end{array}$ & $\begin{array}{l}\text { Persistent apathy } \\
(\mathrm{n}=11)\end{array}$ & p Value* \\
\hline $\begin{array}{l}\text { Change in total Unified Parkinson Disease } \\
\text { Rating Scale motor score } \dagger \text { (SD) }\end{array}$ & $12.9(13.7)$ & $21.5(15.6)$ & $36.8(18.1)$ & 0.001 \\
\hline Change in motor subscore $A+(S D)$ & $8.8(10.8)$ & $14.4(12.0)$ & $24.7(12.9)$ & 0.003 \\
\hline Change in motor subscore $B \S(S D)$ & $2.6(2.6)$ & $5.2(3.7)$ & $7.2(5.3)$ & 0.004 \\
\hline Change in MADRS score (SD) & $-0.2(3.9)$ & $4.3(8.9)$ & $-1.0(1.6)$ & 0.042 \\
\hline Change in MMSE score§ (SD) & $-3.4(4.8)$ & $-8.3(6.6)$ & $-12.2(6.4)$ & $<0.001$ \\
\hline Change in NPI apathy score $\dagger$ (SD) & $-0.10(0.90)$ & $8.3(2.8)$ & $1.2(3.1)$ & $<0.001$ \\
\hline \multicolumn{5}{|l|}{ Depression } \\
\hline Baseline:, n (\%) & $5(17.2)$ & $5(12.8)$ & $6(54.5)$ & 0.055 \\
\hline Follow-up§, n (\%) & $3(10.3)$ & $11(28.2)$ & $5(45.5)$ & 0.004 \\
\hline \multicolumn{5}{|l|}{ Dementia } \\
\hline Baseline§, n (\%) & $2(6.9)$ & $14(35.9)$ & $7(63.6)$ & $<0.001$ \\
\hline Follow-up§, n (\%) & $6(20.7)$ & $34(87.2)$ & $10(90.9)$ & $<0.001$ \\
\hline
\end{tabular}

Data are expressed as mean (SD) or number (percentage of assessed patients); all available data included. All $p$ values are twotailed. A positive change in total motor score and motor subscore A or B represents a worsening of motor performance, negative Montgomery and Aasberg Depression Rating Scale (MADRS) and Neuropsychiatric Inventory (NPI) apathy score changes represent improvement of depressive and apathetic symptoms, whereas negative Mini-Mental State Examination (MMSE) score changes represent a worsening of cognitive symptoms, compared with baseline values.

${ }^{*} p$ values computed using Kruskal-Wallis tests for continuous variables, linear-by-linear association test for categorical variables. $\dagger$ Comparisons: Never apathy versus Incident apathy \& Never apathy versus Persistent apathy \& Incident apathy versus Persistent apathy: $\mathrm{p}<0.05$

\#Comparisons: Never apathy versus Persistent apathy \& Incident apathy versus Persistent apathy: $p<0.05$.

§Comparisons: Never apathy versus Incident apathy \& Never apathy versus Persistent apathy: $p<0.05$ for motor subscore $B$ and depression at follow-up, $p \leqslant 0.01$ for MMSE, $p \leqslant 0.005$ for dementia at baseline, and $p<0.001$ for dementia at follow-up.

ๆComparison: Never apathy versus Incident apathy: $p<0.05$.

model coefficients and the Hosmer-Lemeshow goodness-of-fit test supported our regression models as being valid.

\section{DISCUSSION}

In this longitudinal study of patients derived from a community-based cohort of prevalent PD, apathy was a frequent and persistent behavioural feature. Although the sample size was limited, this finding suggests that apathy may be caused by an irreversible biological deficiency in patients with more advanced $\mathrm{PD}$. The biological basis of apathy is unclear, but neurotransmitter deficits in the dopaminergic, cholinergic, noradrenergic and serotonergic systems have been found to be involved in apathy. ${ }^{22}$ These deficits are also thought to relate to depression and dementia associated with PD (PDD) ${ }^{23}$ This may explain our and previous $^{5}$ observations of apathy being associated with more severe depressive symptoms and greater cognitive impairment.

The occurrence of apathy increased substantially and affected more than $60 \%$ at follow-up, yielding an annual incidence rate of $12.3 \%$. In comparison, apathy (NPI $\geqslant 4$ ) was present in $38 \%$ of a clinic-based sample of patients with PDD drawn from an international cross-sectional multicentre study published recently. ${ }^{24}$ These figures, and previous reports of increased distress in care givers of PD patients with apathy, ${ }^{24}$ highlight apathy as a frequent and important feature in advancing PD.

Dementia and a more rapid decline in speech and axial impairment, features predominately associated with dysfunction in non-dopaminergic subcortical structures, independently predicted incident apathy. Other studies have reported that progression of these motor symptoms is associated with incident dementia and ageing, ${ }^{1125}$ and so the high incidence of apathy in our cohort may have been caused by brain changes related to both normal ageing and the neurodegenerative process of dementia in PD. Although previous studies have shown that dopaminergic treatment may decrease apathy in patients with PD without dementia and major depression, ${ }^{26}$ our findings suggest that dopaminergic dysfunction does not play a major role in the pathophysiology of apathy in more advanced PD with comorbid depression and dementia. It is generally accepted that cognitive impairment in $\mathrm{PD}$ is characterised by subcortical and eventually cortical cholinergic deficits and Lewy body pathology ${ }^{27}$ Recent consensus criteria consider apathy a supporting feature of $\mathrm{PDD},{ }^{28}$ which is clearly emphazised by our finding that nearly $90 \%$ of patients with PDD had clinically significant apathy.

The main strength of this study is the prospective longitudinal design that involved patients derived from a community-based cohort with prevalent PD. Patients were carefully diagnosed as having PD according to published criteria, and in those who underwent autopsy all fulfilled neuropathological criteria for PD. Finally, the neuropsychiatric and neuropsychological assessments were performed blind to the motor evaluation. Limitations include the long time intervals between study visits, and that minor depression and clinical significant apathy were defined by cut-off scores rather than standardised diagnostic criteria. However, currently there are no generally accepted criteria and ad-hoc rating scales for apathy. ${ }^{1}$ The NPI is frequently used to screen and assess the severity of neuropsychiatric symptoms, including apathy, in neurodegenerative disorders and has recently been considered a "suggested" scale for apathy in $\mathrm{PD},{ }^{1}$ with a cut-off $\geqslant 4$ being recommended as indicating clinically significant apathy in $\mathrm{PD} .{ }^{18}$

Our study demonstrates that apathy is a key neuropsychiatric disorder in more severe cases of PD, underlining the need for drug trials to improve motivation, cognition and emotional deficits in patients with PD. Further research in larger groups of patients with early $\mathrm{PD}$ and with more frequent evaluations over time is necessary to identify factors that may predict apathy at an earlier stage of the disease.

Competing interests: None.

Ethics approval: Ethics approval was provided by the Regional Committee for Medical Research Ethics at the University of Bergen, Norway. 
Patient consent: Obtained.

Provenance and peer review: Not commissioned; externally peer reviewed.

\section{REFERENCES}

1. Leentjens $\mathbf{A F}$, Dujardin $\mathrm{K}$, Marsh $\mathrm{L}$, et al. Apathy and anhedonia rating scales in Parkinson's disease: critique and recommendations. Mov Disord 2008;23:2004-14.

2. Bonelli RM, Cummings JL. Frontal-subcortical circuitry and behavior. Dialogues Clin Neurosci 2007:9:141-51.

3. Pluck GC, Brown RG. Apathy in Parkinson's disease. J Neurol Neurosurg Psychiatry 2002; 73:636-42.

4. Aarsland D, Larsen JP, Lim NG, et al. Range of neuropsychiatric disturbances in patients with Parkinson's disease. J Neurol Neurosurg Psychiatry 1999;67:492-6.

5. Starkstein SE, Mayberg HS, Preziosi TJ, et al. Reliability, validity, and clinical correlates of apathy in Parkinson's disease. J Neuropsychiatry Clin Neurosci 1992:4:134-9

6. Pedersen KF, Larsen JP, Alves G, et al. Prevalence and clinical correlates of apathy in Parkinson's disease: A community-based study. Parkinsonism Relat Disord 2009;15:295-9.

7. Tandberg E, Larsen JP, Nessler EG, et al. The epidemiology of Parkinson's disease in the county of Rogaland, Norway. Mov Disord 1995;10:541-9.

8. Aarsland D, Perry R, Brown A, et al. Neuropathology of dementia in Parkinson's disease: a prospective, community-based study. Ann Neurol 2005:58:773-6.

9. Fahn S, Elton RL, Members of the UPDRS Development Committee. Unified Parkinson's Disease Rating Scale. In: Fahn S, Marsden CD, Goldstein M, et al, eds. Recent developments in Parkinson's disease. Florham Park: Macmillan Healthcare Information, 1987:153-63.

10. Hoehn MM, Yahr MD. Parkinsonism: onset, progression and mortality. Neurology 1967; 17:427-42.

11. Levy G, Tang MX, Cote LJ, et al. Motor impairment in PD: relationship to incident dementia and age. Neurology 2000:55:539-44.

12. Gjerstad MD, Boeve B, Wentzel-Larsen T, et al. Occurrence and clinical correlates of REM sleep behaviour disorder in patients with Parkinson's disease over time. J Neurol Neurosurg Psychiatry 2008;79:387-91.

13. American Psychiatric Association. Diagnostic and statistical manual of mental disorders. 3rd rev edn. Washington: American Psychiatric Press, 1987.
14. Mattis S. Dementia rating scale. Odessa: Psychological Assessment Resources, 1988.

15. Folstein MF, Folstein SE, McHugh PR. "Mini-mental state". A practical method for grading the cognitive state of patients for the clinician. J Psychiatr Res 1975;12:18998.

16. Buter TC, van den Hout A, Matthews FE, et al. Dementia and survival in Parkinson disease: a 12-year population study. Neurology 2008;70:1017-22.

17. Cummings JL, Mega M, Gray K, et al. The Neuropsychiatric Inventory: comprehensive assessment of psychopathology in dementia. Neurology 1994:44:2308-14

18. Dubois B, Burn D, Goetz C, et al. Diagnostic procedures for Parkinson's disease dementia: recommendations from the movement disorder society task force. Mov Disord 2007;22:2314-24.

19. Montgomery SA, Asberg M. A new depression scale designed to be sensitive to change. Br J Psychiatry 1979;134:382-9.

20. Starkstein SE, Merello M, Jorge R, et al. A validation study of depressive syndromes in Parkinson's disease. Mov Disord 2008:23:538-46.

21. Marsh L, McDonald WM, Cummings J, et al. Provisional diagnostic criteria for depression in Parkinson's disease: report of an NINDS/NIMH Work Group. Mov Disord 2006;21:148-58.

22. van Reekum R, Stuss DT, Ostrander L. Apathy: why care? J Neuropsychiatry Clin Neurosci 2005:17:7-19.

23. Zgaljardic DJ, Foldi NS, Borod JC. Cognitive and behavioral dysfunction in Parkinson's disease: neurochemical and clinicopathological contributions. J Neural Transm 2004;111:1287-301.

24. Aarsland D, Bronnick K, Ehrt U, et al. Neuropsychiatric symptoms in patients with Parkinson's disease and dementia: frequency, profile and associated care giver stress. J Neurol Neurosurg Psychiatry 2007;78:36-42.

25. Levy G, Louis ED, Cote L, et al. Contribution of aging to the severity of different motor signs in Parkinson disease. Arch Neurol 2005;62:467-72.

26. Czernecki V, Pillon B, Houeto JL, et al. Motivation, reward, and Parkinson's disease: influence of dopatherapy. Neuropsychologia 2002;40:2257-67.

27. Emre M. What causes mental dysfunction in Parkinson's disease? Mov Disord 2003:18(6 Suppl):S63-71.

28. Emre M, Aarsland D, Brown R, et al. Clinical diagnostic criteria for dementia associated with Parkinson's disease. Mov Disord 2007:22:1689-707. 